\title{
Maurizio Luisetti
}

\author{
Francesco Blasi ${ }^{1}$ and Angelo G. Corsico ${ }^{2}$
}

Affiliations: ${ }^{2}$ Dept of Pathophysiology and Transplantation, Università degli Studi di Milano, IRCCS Fondazione Ospedale Maggiore Policlinico Cà Granda Milano, Milan, Italy. ${ }^{2}$ Respiratory Diseases Division Foundation IRCCS “San Matteo" Hospital University of Pavia, Pavia, Italy.

Correspondence: Francesco Blasi, Dept of Pathophysiology and Transplantation, Università degli Studi di Milano, IRCCS Fondazione Ospedale Maggiore Policlinico Cà Granda, F. Sforza 35, Milan 20122, Italy.

E-mail: francesco.blasidunimi.it

On October 20, 2014, Maurizio Luisetti MD, Professor of Respiratory Diseases, University of Pavia, Italy, passed away at his home in Pavia. He was 61 years old. We are all shocked and saddened by the unexpected and untimely death of Maurizio, a good friend, a great researcher and an excellent doctor.

He was a very young researcher when, in 1985, he was appointed Head of the Biochemical and Genetic Laboratory University of Pavia. He was among the founding members of Alpha One International Registry, a member of the Coordinating Committee from 1997 to 1999 and a member of the Council from 2000. In 2010, Maurizio was appointed Head of the Respiratory Diseases Unit of Fondazione IRCCS Policlinico San Matteo Pavia and Professor of Respiratory Diseases, University of Pavia.

Maurizio published excellent papers during his too-brief career (194 indexed in PubMed). His outstanding scientific contributions on the genetics and biochemistry of chronic obstructive pulmonary disease and rare pulmonary diseases mirror his attitude toward research, and have added considerably to our knowledge in the field.

Maurizio, as a man, was knowledgable and witty. Joking with a friend and colleague a few days before his death, Maurizio said he had an ungrateful heart: he knew that it would betray him. But the Maurizio we all knew and loved had a great heart. He lived life to the full, in private as well as at work. He was rightly proud of his wife Anna and their two children, Linda and Giorgio, who have all been so strong throughout their pain. Even during this difficult time, Giorgio passed two exams at the University.

North American rock music was one of Maurizio's loves: he was a Grateful Dead fan, last summer he went to the Rolling Stones concert in Rome, and he often recalled a Bob Dylan concert he went to several years ago with his dear friends and colleagues at that time, Stefano Nava and Luciano Zocchi.

As Division Director, he was esteemed and held in great affection by his colleagues, co-workers and students. He was an older brother as much as the colleague, with the most extensive experience and knowledge, and few found him intimidating. He was truly authoritative, never authoritarian. He was intelligent; always smiling, kind and calm. He was a man of science but also always ready to roll up his sleeves and help. A few weeks ago, Maurizio was asked at the very last minute to give a lecture on $\alpha_{1}$-antitrypsin deficiency at the Italian National Congress of Pulmonology. He illustrated the progress made in the study of the disease by recounting his own involvement in the research, starting with a picture of himself in front of his first poster, presented at the 1987 American Thoracic Society meeting in New Orleans, LA, USA. In hindsight, this summing up of his work had a prophetic ring to it.

Maurizio gave more than he received in life. The outpouring of affection and sadness at his funeral was a poignant testimony to his generosity and popularity, and his funeral in Pavia was attended by colleagues from all over Italy and Europe, as well as friends and patients. Speaking on behalf of $\alpha_{1}$-antitrypsin deficiency patients and their families, one of them expressed the bewilderment and dismay they felt at the loss of such a passionate and dedicated friend, doctor, professor, researcher and scientist.

The newspaper obituary of the students of the University of Pavia School of Medicine read "We remember with affection and gratitude your clear and passionate lectures, and your attention and concern for us during our training on the Respiratory Diseases ward. Your life as a teacher, as a doctor and as a man will not end here, but will go on in our lives as men and as doctors. We are eternally grateful to you. Goodbye, dear Professor." 


\section{Maurizio Luisetti}

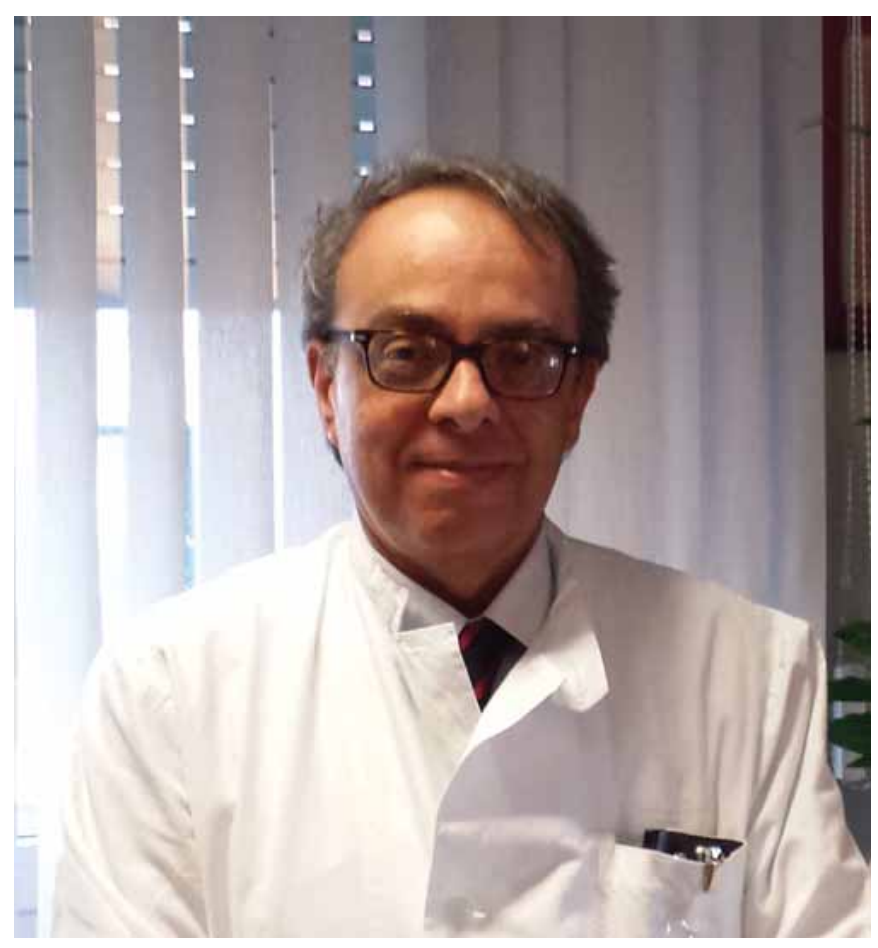

Their words express the sentiments of all of us who were lucky enough to have worked with him. Maurizio, you will remain for us the image of calm engagement in all aspects of life, with your total moral and intellectual integrity.

Ciao, Maurizio! 